\title{
Spin-Polarized STM Investigation of Magnetic Domain Walls
}

\author{
A. Kubetzka, O. Pietzsch, M. Bode, R. Ravlić \\ AND R. WIESENDANGER \\ Institute of Applied Physics, University of Hamburg \\ Jungiusstrasse 11, 20355 Hamburg, Germany
}

\begin{abstract}
The potential of spin-polarized scanning tunneling microscopy and spectroscopy for the investigation of magnetism at the nanometer scale is demonstrated by focusing on magnetic domain walls. After reviewing different measurement modes it is shown that in addition to wall widths and positions the determination of their exact profiles provides further insight into the samples' magnetic properties.
\end{abstract}

PACS numbers: $75.60 . \mathrm{Ch}, 75.75 .+\mathrm{a}, 68.37 . \mathrm{Ef}$

\section{Introduction}

Though the concept of domain walls as the transition regions between magnetic domains in a ferromagnetic sample dates back to the early 1930s [1, 2], domain walls and their properties have attracted renewed attention, due to their decisive role in remagnetization processes. Typical wall widths in $3 d$ bulk ferromagnets are of the order of a hundred nanometers. In ultrathin films and nanostructures, however, which are of technological importance for, e.g., tunneling magnetoresistance (TMR) and giant magnetoresistance (GMR) devices, wall widths can be significantly reduced due to surface and interface anisotropies and are therefore often beyond the resolution limit of standard magnetic imaging techniques such as magnetic force microscopy, Kerr microscopy, or scanning electron microscopy with polarization analysis (SEMPA).

Here we employ spin-polarized scanning tunneling microscopy (SP-STM) to investigate domain walls in two atomic layers thick Fe nanowires prepared on $\mathrm{W}(110)$ as well as on the antiferromagnetic $\mathrm{Cr}(001)$ surface. After reviewing different measurement techniques we discuss the evolution of $360^{\circ}$ domain wall profiles in an external applied field. It can be shown that nonlocal demagnetizing effects are negligible, and that these walls are well described by an exact solution derived 
from a simple $1 \mathrm{D}$ model. On the $\mathrm{Cr}(001)$ surface domain walls originate at screw dislocations due to magnetic frustration. Surprisingly, the wall profiles do not reflect the surface symmetry, which is attributed to a significant magneto-elastic contribution.

\section{Experimental details}

The experiments were performed in UHV systems containing standard surface analysis and preparation facilities with base pressures in the $10^{-11} \mathrm{mbar}$ range. The Fe nanowires were prepared by self-organized (step flow) growth on a W(110) single crystal with an average terrace width of $\approx 25 \mathrm{~nm}$, and investigated with a homemade STM at $T=14 \pm 1 \mathrm{~K}$ [3]. The $\mathrm{Cr}(001)$ single crystal was cleaned by prolonged cycles of $\mathrm{Ar}^{+}$-ion bombardment at elevated temperatures $(T \leq 1100 \mathrm{~K}$ ) and subsequent annealing for 20-30 min at $T=1150 \mathrm{~K}$. The carbon surface contamination could be reduced to below $2 \%$ by using an ion gun equipped with a mass filter (Wien filter). $\mathrm{Cr}(001)$ was investigated at room temperature with a commercial STM.

We used polycrystalline $W$ tips which were electrochemically etched ex situ and flashed in vacuo at $T \geq 2200 \mathrm{~K}$ to remove oxide layers. For spin-resolved studies these tips were coated with 5-10 monolayers (ML) Fe and subsequently annealed at $T=550 \mathrm{~K}$ for $4 \mathrm{~min}$, resulting in a magnetic in-plane sensitivity both at low and room temperature.

\section{Theoretical background}

\subsection{Measurement techniques}

In SP-STM all measurement modes known from conventional STM are available. Since both electrodes, i.e. tip and sample, exhibit a non-vanishing spin-polarization at the Fermi level, the tunnel current is spin-polarized and depends on the electrodes' relative magnetization orientations. It has been shown experimentally for planar tunnel junctions [4] and theoretically for the STM geometry [5] that a simple cosine law is valid, which has already been deduced in Ref. [6] for free electrons in 1D. It can be formulated as

$$
I_{\mathrm{sp}}(r, U)=I_{0}\left[1+\tilde{P}_{\mathrm{eff}}(U) \cos \left(\boldsymbol{M}_{\mathrm{s}}, \boldsymbol{M}_{\mathrm{t}}\right)\right],
$$

where $I_{0}=I_{0}(\boldsymbol{r}, U)$ is the non-spin-polarized part of the tunnel current as a function of tip position $r$ and bias voltage $U$, and $\tilde{P}_{\text {eff }}(U)$ is the bias voltage dependent effective spin-polarization of the tunnel junction. For an electronically homogeneous surface $I_{0}$ and $\tilde{P}_{\text {eff }}$ are independent of the location $r$, and a lateral variation of $I_{\mathrm{sp}}$ can be attributed to a variation of the surface magnetization $M_{\mathrm{s}}(r)$. A similar dependence can be derived for the differential conductance $\mathrm{d} I / \mathrm{d} U[5]$

$$
\frac{\mathrm{d} I}{\mathrm{~d} U_{\mathrm{sp}}}(\boldsymbol{r}, U)=\frac{\mathrm{d} I}{\mathrm{~d} U_{0}}\left[1+P_{\mathrm{eff}}(U) \cos \left(\boldsymbol{M}_{\mathrm{s}}, \boldsymbol{M}_{\mathrm{t}}\right)\right] .
$$


Note that in the general case $\tilde{P}_{\text {eff }}(U)$ and $P_{\text {eff }}(U)$ are not identical, since the spin-polarizations of both electrodes are energy dependent; $\tilde{P}_{\text {eff }}$ denotes the energy integrated polarization in the interval $\left[E_{\mathrm{F}}, E_{\mathrm{F}}+e U\right]$, while $P_{\text {eff }}$ is the polarization at an energy $E_{\mathrm{F}}+e U$.

It follows from Eq. (1) that if the STM is operated in the constant current (CC) mode, a laterally varying $\boldsymbol{M}_{\mathrm{s}}(\boldsymbol{r})$ can be detected as a height variation, and indeed the first SP-STM measurement [7] as well as more recent atomic resolution studies [8,9] were performed in the CC mode. Equation (2) is the basis of spin-polarized spectroscopic techniques. Full $\mathrm{d} I / \mathrm{d} U(U)$ curves can be acquired by stabilizing the tip at specific values of $U_{\text {stab }}$ and $I_{\text {stab }}$, ramping the bias voltage $U$ with the current feedback circuit open while applying a small modulation voltage $U_{\text {mod }}$, and recording the $\mathrm{d} I / \mathrm{d} U$ signal by lock-in technique. From a comparison of two spectra taken above oppositely magnetized domains or before and after the remagnetization of either tip or sample, $P_{\text {eff }}(\mathbf{U})$ can be derived. The extraction of the sample's spin-polarization $P_{\mathrm{s}}(U)$ from $P_{\text {eff }}(U)$ is not a straightforward task, since the tip's spin-polarization $P_{\mathrm{t}}(U)$ has to be known [10]. In an alternative spectroscopic mode the differential conductance at a specific bias voltage, $\mathrm{d} I / \mathrm{d} U(U)$ is recorded simultaneously to the CC image, with the feedback circuit closed. The advantages are reduced measurement times with respect to full spectroscopy and in general an improved signal-to-noise ratio and an apparent separation of the $\mathrm{d} I / \mathrm{d} U(U)$ signal from topographic features acquired in the CC mode.

An example for the three measurement modes discussed above is displayed in Fig. 1. Figure $1 \mathrm{a}$ shows a $\mathrm{d} I / \mathrm{d} U$ map (inset), taken at $I=0.3 \mathrm{nA}$ and $U=-100 \mathrm{mV}$, and a line section across a pair of domain walls. The corresponding
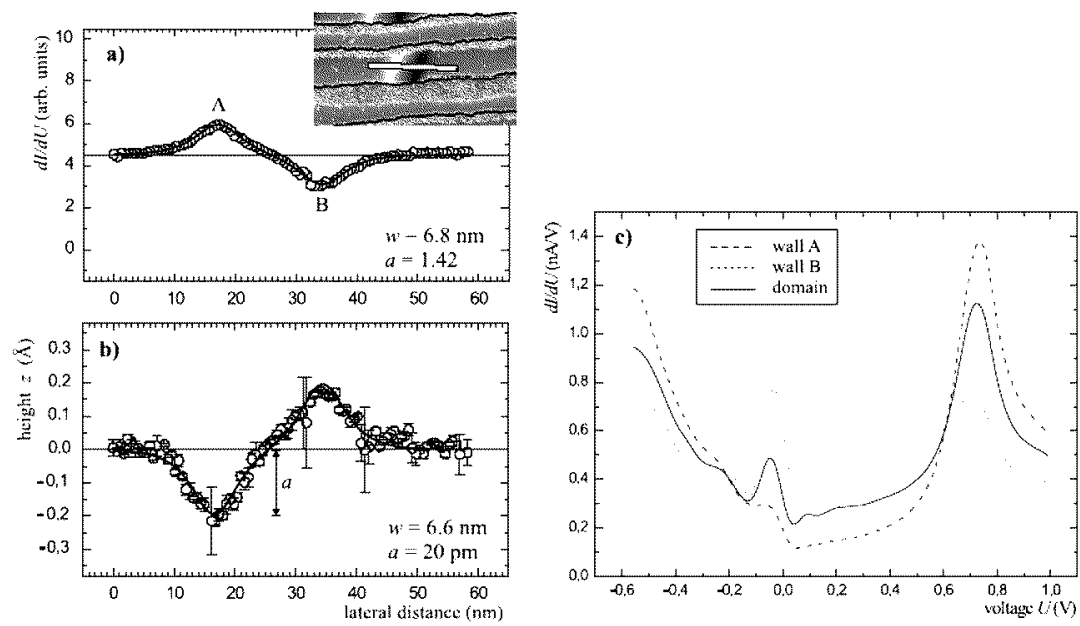

Fig. 1. Comparison of measurement techniques: (a) $\mathrm{d} I / \mathrm{d} U$ map (inset) and line section taken across a pair of domain walls, (b) corresponding height profile, (c) $\mathrm{d} I / \mathrm{d} U$ spectra taken above a domain (solid) and the two oppositely magnetized domain walls $A$ and $B$. 
height profile in Fig. 1b, which was measured simultaneously, has the same characteristics with respect to wall profile and width. A discussion of wall profiles will be given in Sect. 3.2. It should be noted, however, that the $\mathrm{d} I / \mathrm{d} U$ signal in (a) is not independent from the height variation observed in (b). A detailed discussion of this effect can be found in Ref. [10]. Full $\mathrm{d} I / \mathrm{d} U$ spectra taken above the centers of the two domain walls as well as above a domain are displayed in Fig. 1c. The two main features, a relatively weak peak at $U=0.08 \mathrm{~V}$ and a much stronger peak at $U \approx 0.7 \mathrm{~V}$, can be related to $d_{z^{2}}$ states at the $\bar{\Gamma}$ point, and are obviously spin-polarized.

\subsection{Profile of a $180^{\circ}$ wall}

The cosine term in Eq. (2) allows a simple interpretation of $\mathrm{d} I / \mathrm{d} U$ maps imaged with a magnetic tip on an electronically homogeneous surface: the variation of the $\mathrm{d} I / \mathrm{d} U$ signal is proportional to the projection of the local surface magnetization onto the tip magnetization. As an example we consider the one-dimensional case of a single $180^{\circ}$ Bloch wall in a uniaxial medium, which is illustrated schematically in Fig. 2a and described by [11]

$$
\cos (\varphi(x))=\tanh (x / l), \quad l=\sqrt{A / K},
$$

where $\varphi$ is the angle with respect to the easy $z$ axis, and $l$ is the so-called exchange length, which is determined by two material parameters, the exchange stiffness $A$ and the anisotropy constant $K$. It is a characteristic length scale on which a change of the magnetization vector occurs. Though theoretically such a wall is infinitely wide due to its asymptotic behavior, the wall width is usually defined as $w_{0}=2 l$.
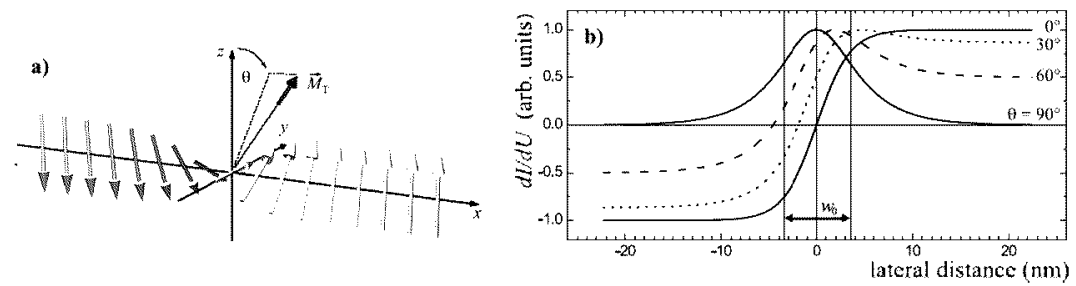

Fig. 2. (a) Schematic representation of a Bloch wall. (b) Wall profiles strongly depend on the tips magnetization $\boldsymbol{M}_{\mathrm{t}}$. The relevant angle $\theta$ is the one between the $z$ direction and the projection of $\boldsymbol{M}_{\mathrm{t}}$ onto the wall plane.

Now, depending on the direction of the tip magnetization $\boldsymbol{M}_{\mathrm{t}}$, different projections of $\boldsymbol{M}_{\mathrm{S}}$ inside the wall are imaged in SP-STM, which is illustrated in Fig. $2 \mathrm{~b}$. With $\boldsymbol{M}_{\mathrm{t}}$ along the sample's easy direction the tanh profile from Eq. (3) is recovered. If $\boldsymbol{M}_{\mathrm{t}}$ is rotated towards the in-plane $y$ direction the profile gradually changes into a $1 / \cosh (x / l)$ function [11]. Note that the relevant angle is not the polar angle of $\boldsymbol{M}_{\mathrm{t}}$, but the angle between the $z$ axis and the projection of $\boldsymbol{M}_{\mathrm{t}}$ onto the wall plane $(y z)$ : With $\boldsymbol{M}_{\mathrm{t}}$ exactly along the $x$ axis the wall would be invisible in SP-STM. 


\section{Results and discussion}

After this preliminary discussion we will now focus on domain walls in real systems.

\section{1. $360^{\circ}$ walls in an external magnetic field}

Since Fe double layer (DL) nanowires on W(110) have a perpendicular magnetic easy axis, $\boldsymbol{M}_{\mathrm{S}}$ has to rotate through an in-plane direction inside a wall, thus in-plane magnetized probe tips are particularly suitable for a study of such domain walls. Figure 3 displays the samples topography and magnetic initial state as a $3 \mathrm{D}$ composite for an Fe coverage $\Theta=1.8 \mathrm{ML}$. Within the DL wires which are separated by narrow regions of ML coverage, two types of $180^{\circ}$ walls can be distinguished by their in-plane magnetization component (see arrows), as we have already seen in Fig. 1. If a perpendicular external magnetic field is applied,

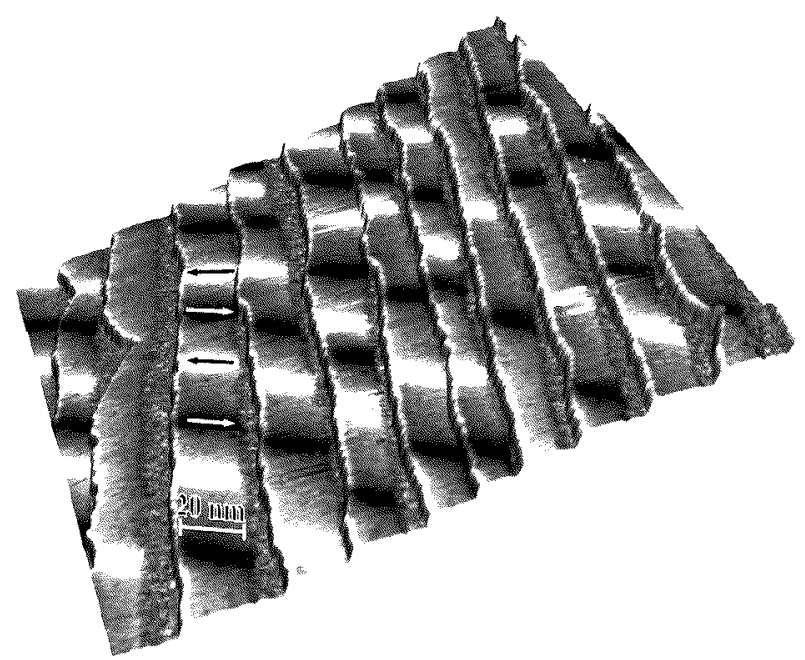

Fig. 3. $200 \times 200 \mathrm{~nm}^{2}$ constant-current (topography) image of $1.8 \mathrm{ML}$ Fe on W(110), colorized with the simultaneously measured $\mathrm{d} I / \mathrm{d} U$ signal, recorded with an in-plane magnetized tip at $U=-0.3 \mathrm{~V}, I=0.3 \mathrm{nA}$, and $T=14 \mathrm{~K}$. Two types of domain walls can be distinguished by their in-plane magnetization component (see arrows).

neighboring pairs of $180^{\circ}$ walls are forced together, which is equivalent to the formation of $360^{\circ}$ walls [12]. These structures are stable up to a critical field value of $B_{\mathrm{c}} \approx 900 \mathrm{mT}$ [13], which is a manifestation of a hard axis anisotropy perpendicular to the rotational plane of the wall [14] ( $x$ direction in Fig. 2a). Its value can be derived from $B_{\mathrm{c}}$ and is of the order of $K_{\mathrm{h}} \approx-2 \times 10^{6} \mathrm{~J} / \mathrm{m}^{3}$ [13]. Here we focus on the evolution of the internal structure of the $360^{\circ}$ walls in fields $B<B_{\mathrm{c}}$. Figure 4 displays $\mathrm{d} I / \mathrm{d} U$ line sections (grey circles) of a single pair of $180^{\circ}$ walls. The area of the inner $180^{\circ}$ rotation between the wall centers has been shaded. As 

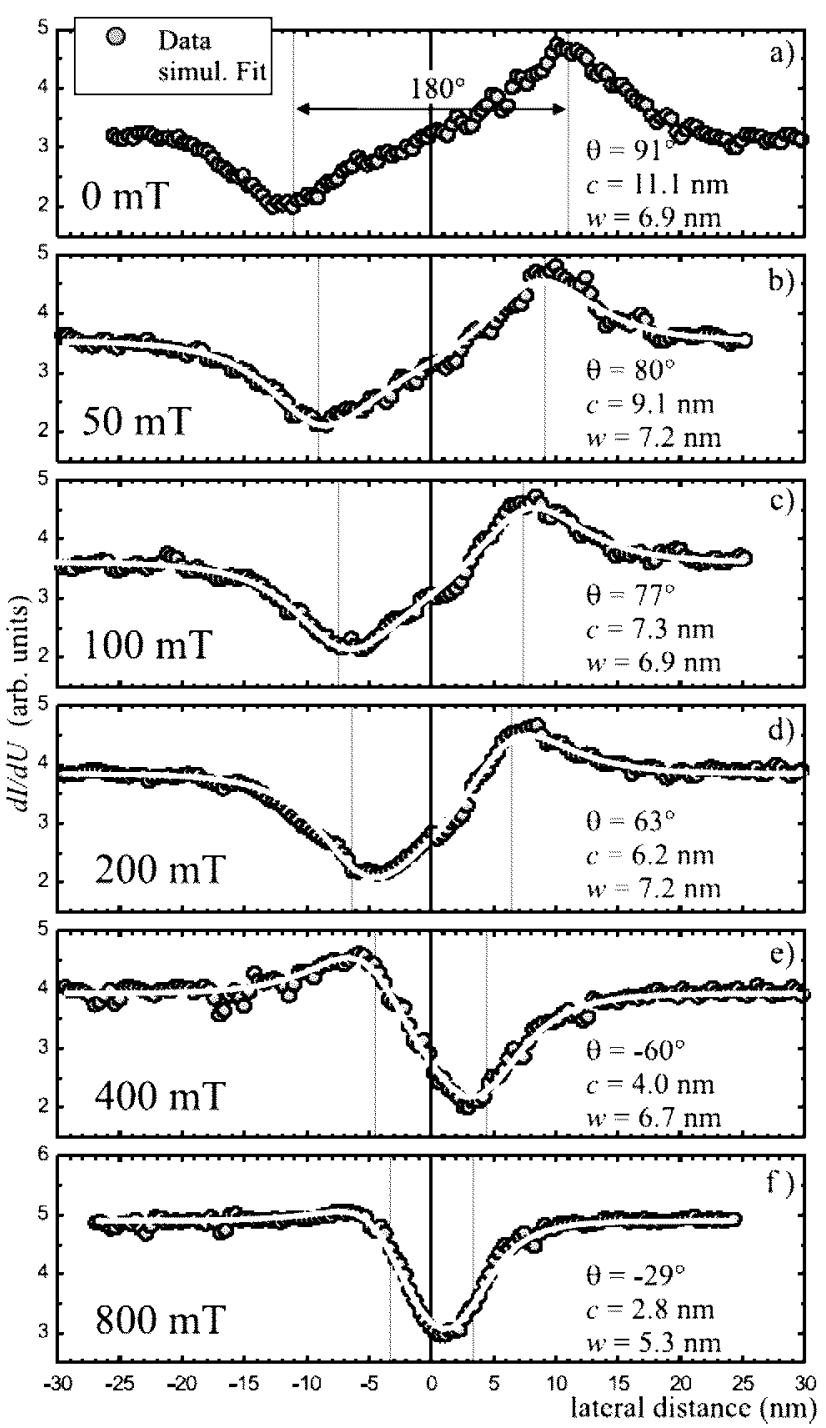

Fig. 4. $\mathrm{d} I / \mathrm{d} U$ line sections (grey circles) across a single $360^{\circ}$ wall in an increasing external field applied along the magnetic easy axis (perpendicular). A simultaneous fit (white lines) is in excellent agreement and allows us to extract the material parameters $A$ and $K_{\text {eff }}$. The shaded areas correspond to the walls' inner $180^{\circ}$ spin rotation between the two opposite in-plane directions.

expected, its lateral extension decreases with increasing field value. It can further be seen that the contrast gradually changes from in-plane to almost perpendicular sensitivity at $800 \mathrm{mT}$, due to an increasing alignment of the tip magnetization $\boldsymbol{M}_{\mathrm{t}}$ along the field direction. 
We compare these results to an exact solution derived by Braun from a simple 1D micromagnetic model [14], which takes into account Zeeman, exchange, and an effective anisotropy energy. The latter includes the crystal anisotropy and the local part of the dipolar (or demagnetizing) energy. Non-local effects are neglected. It can be written as the sum of two standard domain walls of distance $2 c$

$$
\varphi_{360}(x)=\sum_{+,-} \arcsin \left(\tanh \left(\frac{x \pm c}{w / 2}\right)\right)
$$

where the field and material dependent values of $w$ and $c$ are given by

$$
c=\frac{w}{2} \operatorname{arcsinh}\left(\sqrt{\frac{2 K_{\mathrm{eff}}}{M_{\mathrm{s}} B}}\right), \quad w=2 \sqrt{\frac{A}{K_{\mathrm{eff}}+M_{\mathrm{s}} B / 2}} .
$$

For $B \rightarrow 0$ the distance $2 c$ diverges and the zero-field wall width from Eq. (3) is recovered. Taking the varying tip magnetization into account we performed a simultaneous fit to all line sections with

$$
y=y_{0}+a \cos \left(\varphi_{360}(x)+\theta\right),
$$

under the constrains of Eqs. (4) and (5). The resulting curves (white lines) are in excellent agreement with the experimental data, even in the low field regime. The only exception is the zero-field case. Here the equilibrium distance in the experiment depends on the presence of additional neighboring walls and the theoretical model fails, since it considers a single wall pair only. For $B \geq 50 \mathrm{mT}$, however, the agreement implies that the compressing force arising from the Zeeman energy is balanced by an increasing energy penalty due to exchange alone. The non-local part of the dipolar energy, which plays the dominant role in thicker films [11, 14], can be neglected here, due to the reduced dimensions of the system.

As can be seen in Eq. (3), from the zero-field wall width $w_{0}$ only the ratio $A / K$ can be determined. The fitting procedure to the field dependent data, however, allows us to derive two of the three parameters $A, K_{\text {eff }}$, and $M_{\mathrm{s}}$, if one of them is known. Assuming a reasonable value of $M_{\mathrm{s}}=2.0 \times 10^{6} \mathrm{~A} / \mathrm{m}$ for instance, we get $A=1.82 \times 10^{11} \mathrm{~J} / \mathrm{m}$ and $K_{\text {eff }}=1.25 \times 10^{6} \mathrm{~J} / \mathrm{m}^{3}$. Using these parameters as an input to a micromagnetic model of the nanowire system*, it can be concluded that the initial state observed in Fig. 3 is not the magnetic ground state at low temperatures. Roughly speaking, the energy increase due to domain wall formation is not compensated by a reduction of dipolar energy. The high density of domain walls is most likely a result of the dynamics during the cooling process, which is then frozen in a metastable state at low temperatures.

\section{D. Antiferromagnetic surface: $\operatorname{Cr}(001)$}

Bulk Cr exhibits a transversal spin-density wave (SDW) below the Néel transition temperature $T_{\mathrm{N}}=311 \mathrm{~K}$ and a longitudinal SDW below the spin-flip

*We used the Object Oriented Micromagnetic Framework, OOMMF, version 1.2a2, available at http:/math.nist.govóommf/. 
temperature $T_{\mathrm{SF}}=121 \mathrm{~K}$. For the $\mathrm{Cr}(001)$ surface it has been predicted by Blügel et al. [15] that the magnetic moments of any atomically flat terrace couple parallel, but - as a result of the antiferromagnetism of $\mathrm{Cr}$ - adjacent terraces are magnetized antiparallel. This so-called "topological antiferromagnetism" was confirmed experimentally by CC mode SP-STM [7] and more recently by spectroscopic SP-STM [16].

In a ferromagnetic sample magnetic domains and domain walls are expected from a minimization of the dipolar energy. In an antiferromagnet this contribution to the total magnetic energy is zero, since the magnetic moments compensate on an atomic scale. For the $\operatorname{Cr}(001)$ surface, however, it has been shown [17] that domain walls are present due to magnetic frustration at structural imperfections. An example is given in Fig. 5. Panel (a) displays a CC image of the stepped $\mathrm{Cr}(001)$ surface containing two screw dislocations (see arrows). The corresponding

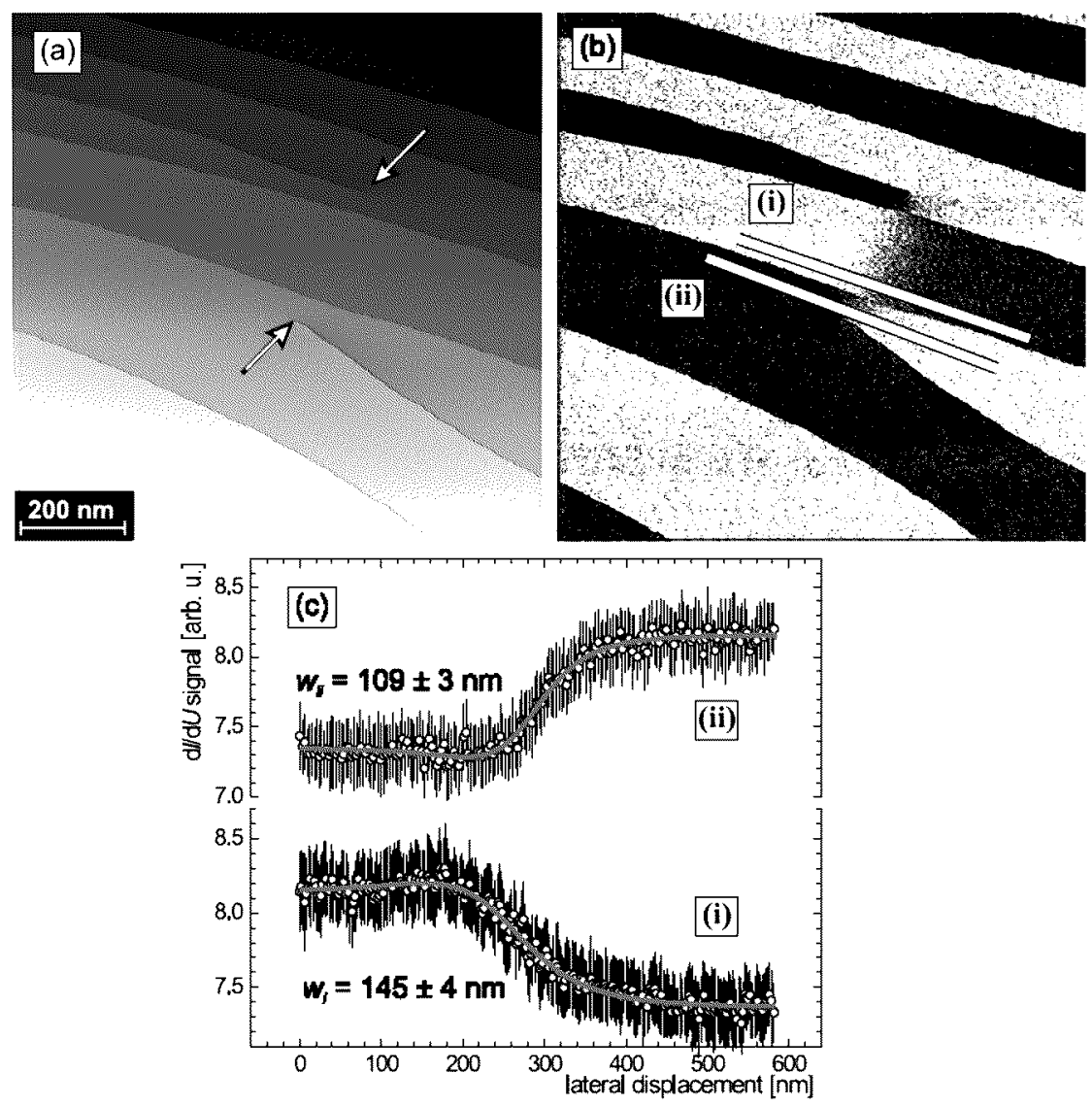

Fig. 5. (a) Topography and (b) magnetic $\mathrm{d} I / \mathrm{d} U$ signal of a $\mathrm{Cr}(001)$ surface with two screw dislocations (measurement parameters: $U=-150 \mathrm{mV}, I=0.7 \mathrm{nA}$ ); (c) Line sections across the domain wall on two adjacent terraces along the lines in (b). 
$\mathrm{d} I / \mathrm{d} U$ map in (b) again confirms the antiparallel ordering of adjacent terraces. More interesting, a $180^{\circ}$ domain wall is present, which starts at one screw dislocation and terminates at the other. The line profiles (i) and (ii) in panel (c) show that for geometrical reasons the wall width decreases close to a dislocation. Far from any screw dislocation wall widths of $w_{\mathrm{Cr}}=150 \pm 10 \mathrm{~nm}$ are measured with profiles as displayed in Fig. 5c, which can be nicely fitted by the standard wall profile from Eq. (3). This is a surprising result, since Eq. (3) is only valid for a medium with uniaxial anisotropy, which is incompatible with the fourfold symmetry of the $\operatorname{Cr}(001)$ surface. From the cubic crystal anisotropy alone a splitting into two $90^{\circ}$ walls is to be expected, which is, however, not the case. We speculate that the observed effective uniaxial anisotropy is caused by the magnetostrictive self-energy. For a rough estimation of the magnetistrictive coefficient $\lambda_{100}$ we assume $A_{\mathrm{Cr}}=1 \times 10^{-11} \mathrm{~J} / \mathrm{m}$ and an anisotropy constant which is dominated by magnetostrictive contributions: $K_{\mathrm{ms}}=\frac{9}{2} \mathrm{C}_{2} \lambda_{100}^{2}$. From $w_{\mathrm{Cr}}=2 \sqrt{A_{\mathrm{Cr}} / K_{\mathrm{ms}}}$ and $C_{2} \approx 1.4 \times 10^{11} \mathrm{~N} / \mathrm{m}^{2}$, we can deduce $\lambda_{100} \approx 10^{-4}-10^{-5}$, which is according to Ref. [11] a rather typical value for magnetostriction.

\section{Summary}

As exemplified in the study of magnetic domain walls in ultrathin $\mathrm{Fe} / \mathrm{W}(110)$ films and at the surface of antiferromagnetic $\operatorname{Cr}(001)$, we have demonstrated the power of SP-STM in the investigation of nanometer scale magnetic structures. Several different magnetic sensitive imaging modes of SP-STM were reviewed. From a detailed analysis of domain wall profiles in bilayer thick Fe nanowires the exchange stiffness parameter $A$ and the effective anisotropy constant $K_{\text {eff }}$ were derived. The results obtained are in excellent agreement with an exact solution of a simple 1D micromagnetic model. On the layered antiferromagnetic Cr surface domain walls appear due to magnetic frustrations arising from structural defects. Contrary to expectations, these walls do not reflect the fourfold symmetry at the surface of this cubic anisotropy material. The observed effective uniaxial anisotropy is tentatively explained by a magnetostrictive self-energy contribution.

\section{Acknowlegments}

Financial support from the DFG (Grant No. Wi1277/19-1 and Graduiertenkolleg "Physik nanostrukturierter Festkörper") is gratefully acknowledged. We thank G. Bihlmayer and H.-B. Braun for stimulating discussions.

\section{References}

[1] F. Bloch, Z. Phys. 74, 295 (1932).

[2] L.D. Landau, E. Lifshitz, Phys. Z. Sowjetunion 8, 153 (1935). 
[3] O. Pietzsch, A. Kubetzka, D. Haude, M. Bode, R. Wiesendanger, Rev. Sci. Instrum. 71, $424(2000)$

[4] T. Miyazaki, N. Tezuka, J. Magn. Magn. Mater. 139, L231 (1995).

[5] D. Wortmann, S. Heinze, Ph. Kurz, G. Bihlmayer, S. Blügel, Phys. Rev. Lett. 86, 4132 (2001).

[6] J.C. Slonzewski, Phys. Rev. B 39, 6995 (1989).

[7] R. Wiesendanger, H.-J. Güntheroth, G. Güntheroth, R.J. Gambino, R. Ruf, Phys. Rev. Lett. 65, 247 (1990).

[8] S. Heinze, M. Bode, A. Kubetzka, O. Pietzsch, X. Nie, S. Blügel, R. Wiesendanger, Science 288, 1805 (2000)

[9] H. Yang, A.R. Smith, M. Prikhodko, W.R.L. Lambrecht, Phys. Rev. Lett. 89, $226101(2002)$

[10] A. Kubetzka, O. Pietzsch, M. Bode, R. Wiesendanger, Appl. Phys. A 76, 873 (2003).

[11] A. Hubert, R. Schäfer, Magnetic Domains, Springer, Berlin (1998).

[12] A. Kubetzka, O. Pietzsch, M. Bode, R. Wiesendanger, Phys. Rev. B 67, 020401 (2003).

[13] A. Kubetzka, M. Bode, O. Pietzsch, R. Wiesendanger, Phys. Rev. Lett. 88, 057201 (2002).

[14] H.-B. Braun, Phys. Rev. B 50, 16485 (1994).

[15] S. Blügel, D. Pescia, P.H. Dederichs, Phys. Rev. B 39, 1392 (1989).

[16] M. Kleiber, M. Bode, R. Ravlić, R. Wiesendanger, Phys. Rev. Lett. 85, 4606 (2000).

[17] R. Ravlić, M. Bode, A. Kubetzka, R. Wiesendanger, Phys. Rev. B 67, 174411 (2003). 